\title{
Diagnostic value of Doppler ultrasound parameters combined with MMP-11 in early breast cancer and benign breast diseases
}

\author{
HUI REN ${ }^{1}$, ZHIPENG SHEN $^{1}$, JIRUI SHEN ${ }^{1}, \mathrm{YU} \mathrm{ZHANG}^{1}$ and YUNHUA ZHANG ${ }^{2}$ \\ ${ }^{1}$ Department of Ultrasound, Liaocheng Third People's Hospital; ${ }^{2}$ Department of Ultrasound, \\ Liaocheng People's Hospital, Liaocheng, Shandong 252000, P.R. China
}

Received November 26, 2019; Accepted December 20, 2019

DOI: $10.3892 / \mathrm{ol} .2020 .11676$

\begin{abstract}
Diagnostic value of Doppler ultrasound parameters combined with matrix metalloproteinase-11 (MMP-11) in early breast cancer and benign breast diseases were investigated. A total of 72 patients who underwent color Doppler ultrasound examination in Liaocheng Third People's Hospital from March 2015 to August 2018 were collected as research subjects, and the blood of 60 healthy subjects who underwent physical examinations was collected. The expression level of MMP-11 in serum of breast cancer patients was evaluated, and the diagnostic value of color Doppler ultrasound combined with MMP-11 in breast cancer was assessed. The diagnostic results of color Doppler ultrasound and the imaging characteristics of breast cancer patients were recorded. The results of biopsy and ultrasound were compared. The expression level of MMP-11 in serum of breast cancer patients was significantly higher than that of healthy subjects $(\mathrm{P}<0.05)$. The AUC of MMP-11 was 0.735 , the sensitivity was $66.67 \%$, and the specificity was $86.11 \%$. Among the 72 patients, there were 41 patients diagnosed with breast cancer by serum MMP-11 examination, 38 patients diagnosed by ultrasound examination, 33 patients diagnosed by combined diagnosis, and 30 patients diagnosed by pathology biopsy. The pathological biopsy was used as the gold standard. The diagnostic efficacy of ultrasound combined with mammography examination was significantly better than the other two single examinations $(\mathrm{P}<0.050)$. In conclusion, Doppler ultrasound parameters combined with MMP-11 has a high diagnostic accordance rate in the diagnosis of breast cancer. At the same time, different diagnostic methods combined with the clinical manifestations of patients can improve the diagnostic accuracy, which is worthy of providing reference and advice for future clinical practice.
\end{abstract}

Correspondence to: Dr Yunhua Zhang, Department of Ultrasound, Liaocheng People's Hospital, 67 Dongchang Xi Road, Dongchangfu, Liaocheng, Shandong 252000, P.R. China

E-mail: ay46y6@163.com

Key words: breast cancer, color Doppler ultrasound, MMP-11, imaging characteristic, diagnostic value

\section{Introduction}

Breast cancer is the most common malignant tumor among women, and its incidence has remained high for many years (1), accounting for $8-12 \%$ of the malignant tumors in the whole body (2). According to the data, there are more than one million cases of breast cancer diagnosed globally every year, and approximately 410,000 deaths (3). Breast cancer is more common in Europe, North America and other developed cities, among which the United States has the highest incidence in the world (4). According to Turner et al (5), in the next 50 years, the incidence of breast cancer will exceed $50 \%$, becoming the second most common malignant tumor after gastric cancer. Furthermore, as early breast cancer has no obvious characteristics, it is often ignored by patients, making them miss the best treatment period, leading to high mortality. The fatality of breast cancer in situ is not high, but once the cancer cells fall off, free cancer cells can be transferred to any place through blood circulation and lymph circulation, and the threat of breast cancer is greatly increased (6). Therefore, 'early detection and early treatment' is advocated for the occurrence of breast cancer in clinical practice, and timely intervention treatment is conducted to ensure the health of patients before in situ cancer has spread (7).

Color Doppler ultrasound is a commonly used method for detection and diagnosis of breast cancer. It can assess the shape, direction, internal structure and edge of lesions from multiple planes, and has high resolution in the fat-dominated breast and the compact gland structure (8). Matrix metalloproteinase (MMP) is a family of zinc endopeptidases which can lyse almost all components of the extracellular matrix and many other soluble and cell-related proteins. In the study of Cheng et al (9), MMP-11 was found as a possible prognostic marker, and the expression reflects the differentiation stage and LNM of breast cancer.

Therefore, by studying the diagnostic value of color Doppler ultrasound parameters combined with MMP-11 in early breast cancer and benign breast diseases and comparing the diagnostic accuracy of single diagnosis of breast cancer, this study provides reference and guidance for clinical practice.

\section{Patients and methods}

Basic patient data. A total of 72 patients underwent color Doppler ultrasound examination in Liaocheng Third People's 
Hospital (Liaocheng, China) from March 2015 to August 2018 and were collected as research subjects, aged 30-65 years, with an average age of $42.73 \pm 12.24$ years. Blood samples were collected from 60 healthy subjects, with an average age of $41.9 \pm 10.3$ years. Both clinical data collection and this study were approved by the medical Ethics Committee of the hospital.

Inclusion and exclusion criteria. Inclusion criteria: Patients whose symptoms were consistent with the clinical manifestations of breast cancer (10). Patients underwent color Doppler ultrasound in the hospital. Female patients. Patients aged 30-70 years, with complete case data. Patients who agreed to cooperate with the arrangement of medical staff in the hospital, and the patient or immediate family member had signed the informed consent.

Exclusion criteria: Patients combined with other tumors. Patients with severe organ failure. Patients with liver and kidney dysfunction. Patients with mental disease. Patients with a history of breast plastic surgery. Patients in pregnancy and lactation. Patients who could not take care of themselves. Patients who were bedridden. Patients transferred to other hospitals. Patients with surgical contraindications.

Blood sample processing. On an empty stomach in the morning, venous blood was extracted and stored at $4^{\circ} \mathrm{C}$ for $30 \mathrm{~min}$. The serum samples were centrifuged at $1,500 \mathrm{x} \mathrm{g}$ and $25^{\circ} \mathrm{C}$ for 10 min to extract the supernatant and stored in a refrigerator at $-80^{\circ} \mathrm{C}$.

Color ultrasound detection and main reagents. Color Doppler ultrasound was performed on subjects using Acuson Sequoia 512 (Siemens AG) with supine position and upper limb lift, fully exposing chest and both axilla. The couplant was applied around the nipple, and the quadrants of the breast were sequentially detected to both axilla. If a lump was found, it was classified according to the number of lactiferous ducts, composition of adipose stroma, and proportion of fibrous glandular tissue in the image under naked eye observation, and the classification standard was referred to (11). enzyme-linked immunosorbent assay (ELISA) was used to determine the expression of MMP-11 in serum. MMP-11 was from Wuhan Fine Biotech Co., Ltd., with the brand of FineTest and article number of EH0782. Operations were in strict accordance with the kit instructions.

Outcome measures. Main outcome measures: the expression level of MMP-11 in serum of breast cancer patients was observed, and the diagnostic value of color doppler ultrasound combined with MMP-11 in breast cancer was observed.

Secondary outcome measures: the diagnostic results of color Doppler ultrasound was observed, and the imaging characteristics of breast cancer patients were evaluated. The results of biopsy and ultrasound were compared.

Statistical method. In this study, SPSS 20.0 software package was used for statistical analysis of the collected data, GraphPad 7 software package was used to draw the required illustrations, and K-S test was used to analyze the distribution of measurement data, in which the normal distribution data was expressed as mean \pm standard deviation $($ mean $\pm \mathrm{SD})$. Independent sample t-test was used for comparison between
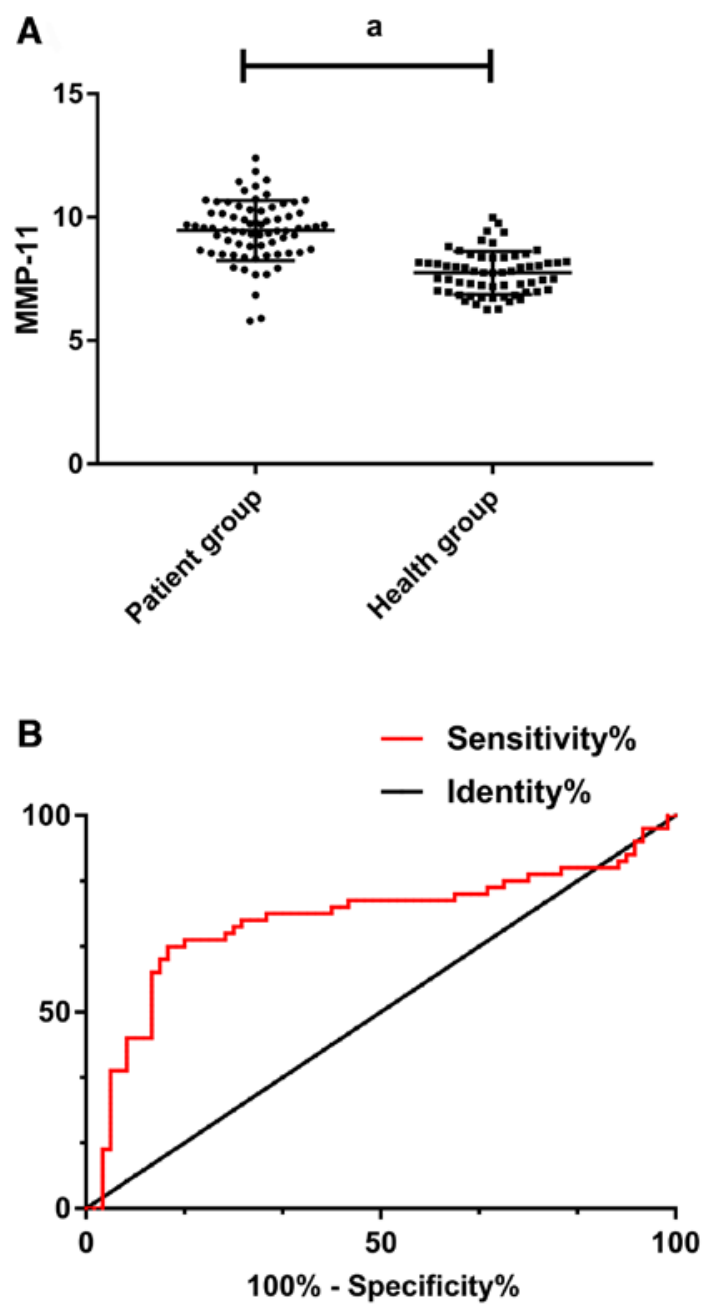

Figure 1. (A) Expression level of MMP-11 was 9.36 \pm 1.25 in breast cancer patients and $7.69 \pm 0.82$ in serum of healthy subjects. Expression level of MMP-11 in serum of breast cancer patients was significantly higher than that of healthy subjects $\left({ }^{\mathrm{a}} \mathrm{P}<0.05\right)$. (B) According to ROC curve analysis, at cut-off value 8.440 , the AUC of MMP-11 was 0.735 , the sensitivity was $66.67 \%$, and the specificity was $86.11 \%(\mathrm{P}<0.05)$. MMP-11, matrix metalloproteinase- 11 .

groups, and paired t-test was used for inter-group comparison analysis. Enumeration data was expressed by (\%), qualified by Chi-square test and presented by $\chi^{2}$ test. ROC was used to plot the diagnostic value of MMP-11 in breast cancer, and $\mathrm{P}<0.05$ indicates a statistical difference.

\section{Results}

Clinical data of patients. The general data of the 72 cases of breast cancer patients, included age, height, ethnicity, education, residence, BMI and fertility circumstance, as shown in Table I.

Expression level and diagnostic value of MMP-11 in serum of breast cancer patients. The expression level of MMP-11 was $9.36 \pm 1.25$ in breast cancer patients and $7.69 \pm 0.82$ in serum of healthy subjects. The expression level of MMP-11 in serum of breast cancer patients was significantly higher than that of healthy subjects $(\mathrm{P}<0.05)$. Furthermore, by plotting ROC, the AUC of MMP-11 was 0.735 , the sensitivity was $66.67 \%$, and the specificity was $86.11 \%, \mathrm{P}<0.05$ (Table II and Fig. 1). 
Table I. Basic patient data.

\begin{tabular}{lc}
\hline Item & {$[\mathrm{n}(\%)]$} \\
\hline Age (years) & \\
$<42$ & $27(37.50)$ \\
$\geq 42$ & $45(62.50)$ \\
Height (cm) & \\
$<155$ & $32(44.44)$ \\
$\geq 155$ & $40(55.56)$ \\
Ethnicity & \\
Han & $52(72.22)$ \\
Others & $20(27.78)$ \\
Education background & \\
$>$ Senior high school & $32(44.44)$ \\
$<$ Senior high school & $40(55.56)$ \\
Residence & \\
City & $55(76.39)$ \\
Countryside & $17(23.61)$ \\
BMI & \\
$<21$ & $31(43.06)$ \\
$\geq 21$ & $41(56.94)$ \\
Fertility circumstance & \\
Multipara & $12(16.67)$ \\
Nullipara & \\
\hline
\end{tabular}

Table II. ROC curve.

MMP-11

\begin{tabular}{lc}
\hline AUC & 0.735 \\
Standard error & 0.048 \\
$95 \%$ CI & $0.641-0.828$ \\
P-value & 0.001 \\
Cut-off & 8.440 \\
Sensitivity [n(\%)] & $66.67 \%$ \\
Specificity (\%) & $86.11 \%$ \\
\hline
\end{tabular}

MMP-11, matrix metalloproteinase-11.

Diagnostic results. Among the 72 patients, there were 41 patients diagnosed with breast cancer by serum MMP-11 examination, 38 patients diagnosed by ultrasound examination, 33 patients diagnosed by combined diagnosis, and 30 patients diagnosed by pathology biopsy. Among the other 42 patients, there were 23 patients diagnosed with adenosis of breast, 10 patients diagnosed with galactocele, 4 patients diagnosed with tuberculosis of breast, and 3 patients diagnosed with mammary duct ectasia.

Imaging characteristics. After the ultrasound diagnosis, all the data of the examination were read by 3 experienced clinicians in the hospital separately and the diagnosis results were jointly given after reaching an agreement. The main manifestations of ultrasound examination in breast cancer patients were:
Table III. Results of MMP-11 in diagnosis of breast cancer.

\begin{tabular}{lccc}
\hline & Biopsy (+) & Biopsy (-) & \\
\hline MMP-11 (+) & 24 & 17 & 41 \\
MMP-11 (-) & 6 & 25 & 31 \\
& 30 & 42 & \\
\hline
\end{tabular}

MMP-11, matrix metalloproteinase-11.

Table IV. Results of ultrasound in diagnosis of breast cancer.

\begin{tabular}{lccc}
\hline & Biopsy (+) & Biopsy (-) & \\
\hline Ultrasound (+) & 26 & 12 & 38 \\
Ultrasound (-) & 4 & 30 & 34 \\
& 30 & 42 & \\
\hline
\end{tabular}

Table V. Results of combined diagnosis of breast cancer.

\begin{tabular}{lccc}
\hline & Biopsy (+) & Biopsy (-) & \\
\hline Combined diagnosis (+) & 29 & 4 & 33 \\
Combined diagnosis (-) & 1 & 38 & 39 \\
& 30 & 42 & \\
\hline
\end{tabular}

i) Unclear boundary and irregular shape, mostly showing burr shape and crab-like shape. ii) Increased anteroposterior diameter of the lesion, and the thickness/length was $>1$. iii) Posterior echo attenuation, multiple medullary cells, loose tissue structure, and no echo enhancement in the lesion posterior area. iv) Visible microscopic calcification within the lesion, with a size of 100-500 $\mu \mathrm{m}$ and acoustic shadowing in the rear. v) Abundant blood flow signals within the lesion, common high speed and high resistance blood flow. The main manifestations of mammography examination were: i) Irregular boundary, high density nodules, uneven density. ii) Burr shape edge, with visible small clusters of shape and gravel like calcification.

Diagnostic efficacy assessment. The pathology biopsy was taken as gold standard. According to the calculation, the sensitivity, specificity and diagnostic accordance rate of MMP-11 for breast cancer were 58.54, 80.65 and $68.06 \%$, respectively. The sensitivity, specificity and diagnostic accordance rate of ultrasound for breast cancer were $68.42,88.24$ and $77.78 \%$, respectively. The sensitivity, specificity and diagnostic accordance rate of combined examination were $87.88,97.44$ and $93.06 \%$, respectively. The diagnostic efficacy of ultrasound combined with mammography examination was significantly better than the two single examinations $(\mathrm{P}<0.050)$ (Tables III-V).

\section{Discussion}

As the most common malignant tumor among women in the world, the incidence and mortality of breast cancer are on the 
rise year by year (12). In order to improve the diagnosis and treatment rate of breast cancer, clinical efforts are being made to explore the pathogenic mechanism of breast cancer from various perspectives for diagnosis and treatment, but no significant breakthrough has been made so far. Poortmans et al (13) considered that the pathogenesis of breast cancer was mainly due to the role of genetic factors, while Tutt et al (14) showed that the pathogenesis of breast cancer was closely related to tumor stem cells. At present, there is no accurate and reliable study that can indicate the exact cause of breast cancer, and the main clinical diagnosis is still imaging. With the development of the disease, increasingly difficult types of breast cancer cannot be effectively determined only through imaging (15). Therefore, it has become a very important research topic to summarize the risk factors of breast cancer for influencing the examination of medical means. Traditional ultrasound and mammography examination is simple, convenient, non-invasive, and has a long history of diagnosis, which is the advantage of breast cancer diagnosis. However, there are controversies about the determination of some benign tumors and cystic hyperplasia of the breast.

MMP-11 belongs to matrix metalloproteins (16), and is closely related to many diseases such as atherosclerosis, rheumatoid arthritis and cancer in process of embryo implantation, organogenesis, tissue degeneration and repair (17-20). MMP-11 typically acts during tissue remodeling that occurs at the epithelial/connective tissue interface to regulate epithelial homeostasis. In cancer, high MMP-11 level in primary tumors are associated with poor prognosis (21-23). In this study, we explored the diagnostic value and accuracy of Doppler ultrasound parameters combined with MMP-11 in early breast cancer and benign breast diseases, so as to provide information for future clinical practice.

In this study, we first observed the expression level of MMP-11 in serum of breast cancer patients, and found that the expression level of MMP-11 in serum of breast cancer patients was significantly higher than that of healthy subjects, indicating that MMP-11 might become a potential target for diagnosis and treatment of breast cancer. ROC curve was drawn and it was found that the AUC of MMP-11 curve was 0.735 , the sensitivity was $66.67 \%$ and specificity was $86.11 \%$, which was not high in sensitivity but high in specificity. This suggests that MMP-11 has certain predictive value for breast cancer patients. Biomarkers are used as tools in cancer diagnosis and treatment stratification. According to Hadler-Olsen et al (24), levels of one or more MMP members were elevated in most cancers. This family of proteolytic enzymes is involved in many stages of cancer development, including angiogenesis, invasion, and metastasis. Therefore, it is expected that MMP can be used as a diagnostic and prognostic marker in cancer patients. Benson et al (25) showed that MMPs are differentially regulated in breast cancer tissues, and they may play different roles in tumor invasion, metastasis and angiogenesis. Therefore, MMPs are of great research value as a diagnostic marker and drug target. The application of ultrasound and MMP-11 single examination has good specificity for breast cancer, but the combined diagnosis of the two is better for the diagnosis of breast cancer. It suggests that the early diagnostic rate of breast cancer can be improved by the combined examination of ultrasonography and mammography. Ultrasound is superior to MMP-11 in the diagnosis of tumor classification, tumor grade, tumor density, breast cystic hyperplasia and benign tumors, which is not available in MMP-11 detection. However, MMP-11 can play different roles in tumor invasion, metastasis and angiogenesis, and can be used as a diagnostic and prognostic marker and drug target. By using the two methods together, they can make up for each other's shortcomings and achieve the best diagnostic effect.

In conclusion, MMP-11 exhibits high expression in breast cancer patients. The ROC curve shows that MMP-11 has a good clinical diagnostic value and the use of ultrasound combined with MMP-11 examination can improve the early diagnostic rate of breast cancer. Moreover, different diagnostic methods combined with the clinical manifestations of patients can improve the diagnostic accuracy, which is worthy of providing reference and advice for future clinical practice.

\section{Acknowledgements}

Not applicable.

\section{Funding}

No funding was received.

\section{Availability of data and materials}

The datasets used and/or analyzed during the current study are available from the corresponding author on reasonable request.

\section{Authors' contributions}

HR conceived the study and drafted the manuscript. ZS and YuZ acquired the data. JS and YunhuaZ analyzed the data and revised the manuscript. All authors read and approved the final manuscript.

\section{Ethics approval and consent to participate}

The study was approved by the Ethics Committee of Liaocheng Third People's Hospital (Liaocheng, China). Patients who participated in this research had complete clinical data. Signed informed consents were obtained from the patients and/or the guardians.

\section{Patient consent for publication}

Not applicable.

\section{Competing interests}

The authors declare that they have no competing interests.

\section{References}

1. DeSantis CE, Fedewa SA, Goding Sauer A, Kramer JL, Smith RA and Jemal A: Breast cancer statistics, 2015: Convergence of incidence rates between black and white women. CA Cancer J Clin 66: 31-42, 2016. 
2. Finn RS, Crown JP, Lang I, Boer K, Bondarenko IM, Kulyk SO, Ettl J, Patel R, Pinter T, Schmidt M, et al: The cyclin-dependent kinase 4/6 inhibitor palbociclib in combination with letrozole versus letrozole alone as first-line treatment of oestrogen receptor-positive, HER2-negative, advanced breast cancer (PALOMA-1/TRIO-18): A randomised phase 2 study. Lancet Oncol 16: 25-35, 2015.

3. Coughlin SS and Ekwueme DU: Breast cancer as a global health concern. Cancer Epidemiol 33: 315-318, 2009.

4. Swain SM, Baselga J, Kim SB, Ro J, Semiglazov V, Campone M, Ciruelos E, Ferrero JM, Schneeweiss A, Heeson S, et al; CLEOPATRA Study Group: Pertuzumab, trastuzumab, and docetaxel in HER2-positive metastatic breast cancer. N Engl J Med 372: 724-734, 2015

5. Turner NC, Ro J, André F, Loi S, Verma S, Iwata H, Harbeck N, Loibl S, Huang Bartlett C, Zhang K, et al; PALOMA3 Study Group: Palbociclib in hormone-receptor-positive advanced breast cancer. N Engl J Med 373: 209-219, 2015.

6. Yu QC, Verheyen EM and Zeng YA: Mammary development and breast cancer: A Wnt perspective. Cancers (Basel) 8: 65, 2016.

7. Collignon J, Lousberg L, Schroeder H and Jerusalem G: Triple-negative breast cancer: Treatment challenges and solutions. Breast Cancer (Dove Med Press) 8: 93-107, 2016.

8. Guo R, Lu G, Qin B and Fei B: Ultrasound imaging technologies for breast cancer detection and management: A Review. Ultrasound Med Biol 44: 37-70, 2018.

9. Cheng CW, Yu JC, Wang HW, Huang CS, Shieh JC, Fu YP, Chang CW, Wu PE and Shen CY: The clinical implications of MMP-11 and CK-20 expression in human breast cancer. Clin Chim Acta 411: 234-241, 2010.

10. Clark A and Fallowfield LP: Breast cancer. CRC Press, 2014

11. Tadayyon H, Sadeghi-Naini A, Wirtzfeld L, Wright FC and Czarnota G: Quantitative ultrasound characterization of locally advanced breast cancer by estimation of its scatterer properties. Med Phys 41: 012903, 2014.

12. Jung KW, Won YJ, Oh CM, Kong HJ, Lee DH and Lee KH; Community of population-based Regional cancer registries: Cancer statistics in Korea: Incidence, mortality, survival, and prevalence in 2014. Cancer Res Treat 49: 292-305, 2017.

13. Poortmans PM, Collette S, Kirkove C, Van Limbergen E, Budach V, Struikmans H, Collette L, Fourquet A, Maingon P, Valli M, et al; EORTC Radiation Oncology and Breast Cancer Groups: Internal mammary and medial supraclavicular irradiation in breast cancer. N Engl J Med 373: 317-327, 2015.

14. Tutt A, Ellis P, Kilburn L, Gilett C, Pinder S and Abraham J: Abstract S3-01: the TNT trial: A randomized phase III trial of carboplatin (C) compared with docetaxel (D) for patients with metastatic or recurrent locally advanced triple negative or BRCA1/2 breast cancer (CRUK/07/012). Cancer Res 75 (Suppl 9): S3-S4, 2015.
15. Basset P, Bellocq JP, Lefebvre O, Noël A, Chenard MP, Wolf C, Anglard P and Rio MC: Stromelysin-3: A paradigm for stroma-derived factors implicated in carcinoma progression. Crit Rev Oncol Hematol 26: 43-53, 1997.

16. Bianchini G, Balko JM, Mayer IA, Sanders ME and Gianni L: Triple-negative breast cancer: Challenges and opportunities of a heterogeneous disease. Nat Rev Clin Oncol 13: 674-690, 2016.

17. Basset P, Bellocq JP, Wolf C, Stoll I, Hutin P, Limacher JM, Podhajcer OL, Chenard MP, Rio MC and Chambon P: A novel metalloproteinase gene specifically expressed in stromal cells of breast carcinomas. Nature 348: 699-704, 1990.

18. Okada A, Saez S, Misumi Y and Basset P: Rat stromelysin 3: cDNA cloning from healing skin wound, activation by furin and expression in rat tissues. Gene 185: 187-193, 1997.

19. Tan J, Buache E, Alpy F, Daguenet E, Tomasetto CL, Ren GS and Rio MC: Stromal matrix metalloproteinase-11 is involved in the mammary gland postnatal development. Oncogene 33: 4050-4059, 2014

20. Schönbeck U, Mach F, Sukhova GK, Atkinson E, Levesque E, Herman M, Graber P, Basset P and Libby P: Expression of stromelysin-3 in atherosclerotic lesions: Regulation via CD40-CD40 ligand signaling in vitro and in vivo. J Exp Med 189: 843-853, 1999.

21. Andarawewa KL, Motrescu ER, Chenard MP, Gansmuller A, Stoll I, Tomasetto C and Rio MC: Stromelysin-3 is a potent negative regulator of adipogenesis participating to cancer cell-adipocyte interaction/crosstalk at the tumor invasive front. Cancer Res 65 : 10862-10871, 2005

22. Motrescu ER and Rio MC: Cancer cells, adipocytes and matrix metalloproteinase 11: A vicious tumor progression cycle. Biol Chem 389: 1037-1041, 2008

23. Tan J, Buache E, Chenard MP, Dali-Youcef $\mathrm{N}$ and Rio MC: Adipocyte is a non-trivial, dynamic partner of breast cancer cells. Int J Dev Biol 55: 851-859, 2011.

24. Hadler-Olsen E, Winberg JO and Uhlin-Hansen L: Matrix metalloproteinases in cancer: Their value as diagnostic and prognostic markers and therapeutic targets. Tumour Biol 34: 2041-2051, 2013.

25. Benson CS, Babu SD, Radhakrishna S, Selvamurugan N and Ravi Sankar B: Expression of matrix metalloproteinases in human breast cancer tissues. Dis Markers 34: 395-405, 2013.

This work is licensed under a Creative Commons Attribution-NonCommercial-NoDerivatives 4.0 International (CC BY-NC-ND 4.0) License. 